\title{
Keppra a magical antiepileptic medication
}

\author{
Shannon Beierle, Pavis Laengvejkal MD
}

\section{Case Presentation}

A 42-yr-old previously healthy man is admitted to the ICU after sustaining a traumatic brain injury from a motor vehicle crash. Patient lost awareness for several moments after the accident. MRI of the head showed an area of hemorrhagic contusion in the left frontal cortex with small amount of hemorrhage in the subarachnoid space. CT scan of the cervical spine showed a flexion "tear-drop" type fracture of C4 with mild posterior displacement of the vertebral body with minimal compression of the cord. Considering the risk of acute seizures in this case and the potential worsening of the spinal cord compression, is levetiracetam (brand name Keppra) an appropriate drug for this patient?

\section{Discussion}

Keppra, or levetiracetam (LEV), is a pyrrolidine derivative anticonvulsant that is chemically unrelated to other available antiepileptic drugs. ${ }^{1}$ Keppra has been used as a broad spectrum anti-epileptic drug (AED). It is FDA approved for use in various types of partial and generalized seizures but its use in acute seizures and status epilepticus was never formally studied. The approved indications include being used in tablet form for the adjunct treatment of adults with partial onset seizures (Dec. 1999), as an injection for adults with partial onset seizure (July 2006), for the adjunct treatment of myoclonic seizures in adults and children 12 years of age and older with

Corresponding author: Pavis Laengvejkal MD Contact Information: pavis.laengvejkal@ttuhsc.edu DOI: 10.12746/swrccc2013.0102.016 juvenile myoclonic epilepsy (Aug. 2006), for adjunct treatment of primary generalized tonic-clonic seizures in adults and children six years of age and older with idiopathic generalized epilepsy (March 2007), for adjunct treatment of primary generalized tonic-clonic seizures in adults and adolescents 16 years of age and older (May 2008), and for adjunctive treatment of partial onset seizures in pediatric patients one month of age or older (Dec. 2011). Because of its broad efficacy, ease of use and favorable side effect profile, the use of levetiracetam has expanded over the years and now includes a number of clinical situations beyond the original FDA approved indications for the drug.

\section{Structure and mechanism of action.}

Levetiracetam is structurally unrelated to other currently available anticonvulsants and the exact mechanism of action is unknown. ${ }^{1}$ The unique antiepileptic effects of levetiracetam appear to involve the following: LEV binds to synaptic vesicle protein 2A (SV2A). It appears to act as a modulator of synaptic vesicle exocytosis, leading to direct inhibition of presynaptic neurotransmitter release in a use dependent fashion. ${ }^{2}$ It also reduces the release of calcium from intraneuronal stores, which leads to changes in the levels of intraneuronal calcium. ${ }^{3}$ LEV also partially inhibits N-type calcium channels, which also leads to changes in the levels of intraneuronal calcium. ${ }^{4}$ Additionally, it opposes the activity of the negative allosteric modulators zinc and ß-carbolines on GABA- and glycin-gated currents. However, LEV does not display conventional GABA enhancing activity. It also stabilizes altered (dysfunctional) human GABA A receptors in the epileptic brain in a use-dependent fashion. Finally, it appears to inhibit excessive synchronized activity between neurons by preventing burst firing of 
neurons without affecting normal neuronal excitability. This may prevent excessive synchronization of epileptiform burst firing and propagation of seizure activity.

\section{Pharmacokinetic Properties}

LEV is rapidly and almost completely absorbed after oral administration, with a relative bioavailability of $100 \% .^{7}$ This is a very desirable feature of LEV that is not share by most other antiepileptic medications. In the adult, the pharmacokinetics of a single oral dose of LEV are predictable, linear, dose proportional, and time independent with the steady state being reached in 48 hours.

The time to Cmax is about one hour without regard to meals. The distribution is close to that of total body water volume. The drug's protein binding is less than $10 \%$, in contrast to other drugs used in the treatment of status epilepticus such as phenytoin and valproic acid that are more than $90 \%$ bound to serum proteins. For this reason, clinically significant interactions between LEV and other drugs through competition for protein binding sites are unlikely.

The major metabolic pathway is enzymatic hydrolysis of the acetamide group, which produces the major carboxylic acid metabolite ucb L057. Metabolism is not dependent on any hepatic cytochrome $\mathrm{P} 450$ isoenzyme (CYP), LEV is eliminated by renal excretion. About $66 \%$ of the administered dose is excreted as the unchanged drug. The mechanism of excretion is glomerular filtration with subsequent partial tubular reabsorption. The metabolite ucb L057 is excreted by glomerular filtration and active tubular secretion. The plasma half-life in adults is about 7-8 hours and is unaffected by dose, route of administration, or repeated administration.

\section{Potential drug interactions}

LEV has infrequent pharmacokinetic interactions with AEDs or other medications. ${ }^{1}$ In a pooled analysis of placebo-controlled trials in adult pa- tients with partial onset seizures receiving adjunctive LEV, there was no evidence of pharmacokinetic drug interactions between LEV and other AEDs (carbamazepine, clobazam, clonazepam, diazepam, gabapentin,lamotrigine, oxcarbazepine, phenobarbital, phenytoin, primidone, topiramate, valproic acid and vigabatrin). ${ }^{8,9}$ In contrast to other anticonvulsants such as phenytoin, carbamazepine and valproic acid, there is no evidence of interactions between LEV and digoxin, warfarin, or low-dose oral contraceptives in healthy volunteers.

\section{Current data onlevetiracetamuse in seizure patients}

Data currently exists to support both FDA approved as well as a handful of off-label uses for LEV. LEV was found to significantly reduce the frequency of partial onset seizures in adult patients when administered in combination with other AEDs. ${ }^{9}$ A noninferiority trial assessed the efficacy of LEV relative to twice-daily carbamazepine controlled release (CR) in patients 16 years or older with newly diagnosed epilepsy who had experienced two or more partial onset or GTC seizures in the previous year. It showed that treatment with LEV monotherapy was not inferior to treatment with carbamazepine CR monotherapy in these patients. ${ }^{10}$ The use of LEV as adjunctive therapy in the treatment of patients greater than 12 years and less than 65-years-old with myoclonic seizures associated with refractory idiopathic generalized epilepsy was evaluated in a placebo-controlled trial. Data showed that LEV was effective in controlling myoclonic seizures in these patients. It also showed that LEV was associated with control of other seizure types (e.g., absences and GTC seizures) often associated with myoclonic seizures. ${ }^{11}$ Additionally, a placebo-controlled trial evaluated the use of LEV as adjunctive therapy in patients aged 4-65-years-old with GTC seizures associated with idiopathic generalized epilepsy and data showed adjunctive LEV was associated with significantly better seizure control than placebo. ${ }^{12}$

The evidence for early use of LEV in the treatment of status epilepticus is limited. The efficacy reported in the early retrospective reports was better than expected and some feared it was due to pub- 
lication bias and recommended caution when using these data in clinical decision-making. ${ }^{13}$

In a prospective, single-center, randomized, single-blinded comparative trial of LEV versus phenytoin (2:1 ratio) in patients with severe traumatic brain injury or subarachnoid hemorrhage, patients received an IV load with either LEV or fosphenytoin followed by standard IV doses of LEV or phenytoin. Doses were adjusted if the patient had a seizure and to maintain therapeutic serum phenytoin concentrations. Continuous EEG monitoring was performed for the initial 72 hours; outcome data were then collected. This study showed improved long-term outcomes of LEV-treated patients vs. phenytoin-treated patients. LEV appears to be an alternative to phenytoin for seizure prophylaxis in this setting. ${ }^{14,15}$

A prospective evaluation was conducted of a series of cases consisting of patients over the age of 60 years who had suffered a stroke and had at least one epileptic seizure in the late post-stroke phase (more than two weeks). Patients began treatment with LEV monotherapy, underwent check-ups at one and six months of treatment, and the effectiveness and safety of the drug were evaluated. Data showed that LEV in monotherapy can be a safe, effective therapeutic option for elderly patients who have presented with epilepsy following a stroke. ${ }^{16}$

One case series analysis evaluated the effects of LEV monotherapy on brain tumor related patients, adverse events, cognitive functioning and quality of life in patients with epilepsy secondary to a brain tumor. Data indicate that LEV use in these patients is safe and efficacious and has apositive impact on quality of life. ${ }^{17}$

\section{Doses for Keppra}

1. For adjunctive treatment of partial seizure, initially $500 \mathrm{mg}$ IV twice daily. Titrate as needed by $1000 \mathrm{mg}$ per day IV every two weeks not to exceed $3000 \mathrm{mg}$ IV per day. When converting to PO, use the same doses and frequencies as IV doses.

2. For adjunctive treatment of myoclonic seizure and primary generalized tonic clonic seizure, the rec- ommended dose is $3000 \mathrm{mg} P O$ per day but start with $500 \mathrm{mg} P O$ twice daily and titrate by $1000 \mathrm{mg}$ per day PO every two weeks to the recommended dose of 3000 mg PO daily.

3. For seizure prevention in traumatic brain injury and post-stroke patients, start with $500 \mathrm{mg}$ IV twice daily. Then titrate upward by $1000 \mathrm{mg}$ per day every two weeks up to $3000 \mathrm{mg}$ per day as needed.

\section{Adverse effects}

During clinical trials of levetiracetam in the adjunct treatment of all seizure types studied in adult or pediatric patients four years of age and older, the following centrally-mediated effects were reported more frequently in patients receiving levetiracetam than placebo: headache (14\% vs. $13 \%)$, dizziness (5$9 \%$ vs. $2-4 \%$ ), ataxia ( $3 \%$ vs. $1 \%$ ), vertigo (3-5\% vs. $1-3 \%)$, paresthesias ( $2 \%$ vs. $1 \%)$, hyperreflexia $(2 \%$ vs. $1 \%)$, fatigue $(10 \%$ vs. $8 \%$ ), and drowsiness (12$23 \%$ vs. $2-11 \%$ ). Adverse reactions to LEV are similar between oral and parenteral formulations. Since LEV is used as adjunctive therapy, the incidence of adverse reactions directly attributable to the drug cannot be adequately determined. ${ }^{18,19}$

\section{Case scenario}

Our patient would benefit from seizure prophylaxis, and levetiracetam appears to be a suitable medication to use. ${ }^{14,15}$

\section{KEYPOINTS}

1. While the exact mechanism of action for levetiracetam remains unknown, it appears to have minimal side effects and drug interactions.

2. While only FDA approved for 6 uses, there is a substantial body of literature to support use in other seizure disorders.

3. There is a need for more prospective studies to evaluate the potential benefits of using levetiracetam for seizure prophylaxis in settings other than epilepsy. 


\section{Key words}

Keppra, levetiracetam, seizures, anti-epileptic drugs, status epilepticus, ICU, post-traumatic seizures

Author Affiliation: Shannon Beierle is a medical student in the SOM at TTUHSC. Pavis Laengvejkal is a resident in internal medicine and neurology at TTUHSC.

Received: $12 / 30 / 2012$

Accepted: 03/24/2013

Reviewers: John Detoledo MD, Kenneth Nugent MD

Published electronically: 04/15/2013

Conflict of Interest Disclosures: None

\section{REFERENCES}

1. Kaminski, R.M., et al., Benefit of combination therapy in epilepsy: a review of the preclinical evidence with levetiracetam. Epilepsia, 2009. 50(3): p. 387-97.

2. Vogl, C., et al., The synaptic vesicle glycoprotein 2A ligand levetiracetam inhibits presynaptic $\mathrm{Ca} 2+$ channels through an intracellular pathway. Mol Pharmacol, 2012. 82(2): p. 199-208.

3. Nagarkatti, N., L.S. Deshpande, and R.J. DeLorenzo, Levetiracetam inhibits both ryanodine and IP3 receptor activated calcium induced calcium release in hippocampal neurons in culture. Neurosci Lett, 2008. 436(3): p. 289-93.

4. Lukyanetz, E.A., V.M. Shkryl, and P.G. Kostyuk, Selective blockade of N-type calcium channels by levetiracetam. Epilepsia, 2002. 43(1): p. 9-18.

5. Rigo, J.M., et al., The anti-epileptic drug levetiracetam reverses the inhibition by negative allosteric modulators of neuronal GABA- and glycine-gated currents. Br J Pharmacol, 2002. 136(5): p. 659-72.

6. Niespodziany, I., H. Klitgaard, and D.G. Margineanu, Desynchronizing effect of levetiracetam on epileptiform responses in rat hippocampal slices. Neuroreport, 2003. 14(9): p. 1273-6.
7. Patsalos, P.N., Clinical pharmacokinetics of levetiracetam. Clin Pharmacokinet, 2004. 43(11): p. 707-24.

8. Gidal, B.E., et al., Effect of levetiracetam on the pharmacokinetics of adjunctive antiepileptic drugs: a pooled analysis of data from randomized clinical trials. Epilepsy Res, 2005. 64(1-2): p. $1-11$.

9. Shorvon, S.D., et al., Multicenter double-blind, randomized, placebo-controlled trial of levetiracetam as add-on therapy in patients with refractory partial seizures. European Levetiracetam Study Group. Epilepsia, 2000. 41(9): p. 1179-86.

10. Brodie, M.J., et al., Comparison of levetiracetam and controlled-release carbamazepine in newly diagnosed epilepsy. $\mathrm{Neu}$ rology, 2007. 68(6): p. 402-8.

11. Noachtar, S., et al., Levetiracetam for the treatment of idiopathic generalized epilepsy with myoclonic seizures. Neurology, 2008. 70(8): p. 607-16.

12. Berkovic, S.F., et al., Placebo-controlled study of levetiracetam in idiopathic generalized epilepsy. Neurology, 2007. 69(18): p. 1751-60.

13. Zelano, J. and E. Kumlien, Levetiracetam as alternative stage two antiepileptic drug in status epilepticus: a systematic review. Seizure, 2012. 21(4): p. 233-6.

14. Jones, K.E., et al., Levetiracetam versus phenytoin for seizure prophylaxis in severe traumatic brain injury. Neurosurg Focus, 2008. 25(4): p. E3.

15. Szaflarski, J.P., et al., Prospective, randomized, single-blinded comparative trial of intravenous levetiracetam versus phenytoin for seizure prophylaxis. Neurocrit Care, 2010. 12(2): p. 165-72.

16. Magomedova, A., et al., [The results of the use of levetiracetam (keppra) in post-stroke epilepsy]. Zh Nevrol Psikhiatr Im S S Korsakova, 2012. 112(6 Pt 2): p. 40-4.

17. Maschio, M., et al., Levetiracetam monotherapy in patients with brain tumor-related epilepsy: seizure control, safety, and quality of life. J Neurooncol, 2011. 104(1): p. 205-14.

18. Steinhoff, B.J., et al., The SKATE study: an open-label community-based study of levetiracetam as add-on therapy for adults with uncontrolled partial epilepsy. Epilepsy Res, 2007. 76(1): p. 6-14.

19. Morrell, M.J., et al., The KEEPER trial: levetiracetam adjunctive treatment of partial-onset seizures in an open-label community-based study. Epilepsy Res, 2003. 54(2-3): p. 153-61. 